\title{
Artemisia Naphta: A novel oil extract for sensitive and acne prone skin
}

\section{Kan Tao', Lili Guo ${ }^{1}$, Jose Fernandez², Corey Webb², Junfeng $\mathrm{Liu}^{3}$, Xincheng Hu${ }^{1}$, Dan Yang ${ }^{1}$ and Edwardo Perez ${ }^{2 *}$}

\author{
${ }^{1}$ Shanghai Chicmax Cosmetic Co, Ltd, Floor 38, Global Harbor B, No 3300 North Zhongshan \\ Road, Putuo District, Shanghai, China \\ ${ }^{2}$ Signum Biosciences Ltd, 11 Deer Park Drive, Suite 202 Monmouth Junction, New Jersey, USA \\ ${ }^{3}$ Shanghai Kunyao Biotechnology Co, Ltd, Floor 25, Global Harbor B, No 3300 North Zhongshan \\ Road, Putuo District, Shanghai, China
}

\section{Abstract}

Background: The plant Artemisia annua has been used in traditional Chinese medicine for many years. Rich in bioactive molecules, the $A$. annua plant is used to extract the anti-malaria compound artemisinin $(<1 \%)$, which results in most of the plant being unutilized. One byproduct of artemisinin extraction is artemisia naphtha (AN), which has yet to be studied extensively.

Aims: Study the activity of a novel AN oil extract against microbes, pro-inflammatory cytokines, and dermatological endpoints that are key for eczema and acne pathogenesis to determine if an effective $A$. annua extract for these skin conditions can be developed.

Methods: Gas chromatography-mass spectrometry was performed to determine the composition of AN oil. P. acnes, S. aureus, M. furfur, and C. albicans were cultured to determine minimal inhibitory concentration. In vitro studies utilizing keratinocytes and macrophages were treated with AN oil and gene expression measured by quantitative RT-PCR. A 13-subject clinical trial was performed with $1 \%$ AN oil Gel to assess its potential benefits for sensitive and acne prone skin.

Results: AN oil upregulates filaggrin gene expression and possesses antimicrobial and antiinflammatory activity inhibiting LPS, S. aureus and "Th2-induced" pro-inflammatory mediator release (IL-6, IL-8 and TSLP). Clinical assessment of $1 \%$ AN Gel shows it reduces acne blemishes and the appearance of redness.

Conclusion: Previously an underutilized and unpurified byproduct, AN is now the source to develop the first topical AN oil for cosmetic use with an activity profile that suggests it is effective for those with sensitive and/or acne prone skin.

\section{More Information}

*Address for Correspondence: Edwardo Perez, Signum Biosciences Ltd, 11 Deer Park Drive, Suite 202 Monmouth Junction, New Jersey, USA, Tel: 908-400-3990; 732.329.6344; Fax 732.329.8344;

Email: eperez@signumbio.com

Submitted: June 05, 2021

Approved: June 14, 2021

Published: June 15, 2021

How to cite this article: Tao K, Guo L,

Fernandez J, Webb C, Liu J, et al. Artemisia Naphta: A novel oil extract for sensitive and acne prone skin. Ann Dermatol Res. 2021; 5: 022-029.

DOI: 10.29328/journal.adr.1001018

Copyright: @ 2021 Tao K, et al. This is an open access article distributed under the Creative Commons Attribution License, which permits unrestricted use, distribution, and reproduction in any medium, provided the original work is properly cited.

Keywords: Anti-inflammatory; Antimicrobial; Acne; Eczema; Sensitive skin; Cosmetic

(T) Check for updates

(ึ) open Access

\section{Introduction}

Artemisia annua (A. annua) is an annual herb used in traditional Chinese medicine as a remedy for malaria and fever [1]. The anti-malarial activity is derived from artemisinin, which has also been shown to have anti-viral, anti-cancer, antifibrotic, anti-inflammatory, and anti-bacterial properties [2]. Artemisinin [3], its derivatives [4] and A. annua extracts rich in flavonoids [5] have also been shown to reduce inflammation and contact sensitization when applied topically to skin. Extraction of artemisinin from $A$. annua yields only small amounts of the bioactive molecule ( $\sim 0.1$ to $0.7 \%$ ) [6], resulting in much of the $A$. annua extract and many of its other bioactive molecules [2] going unused. A recent report shows that $A$. annua extracts reduce redness, inflammation and improve skin barrier repair [7]. Due to Chinese regulations banning the use of artemisinin in cosmetics, we sought to develop a novel topical A. annua extract from current artemisinin extract byproducts that would work to simultaneously improve the extraction's environmental footprint, and potentially provide several benefits to the skin.

One byproduct from artemisinin extraction from A. annua powder is artemisia naphtha (AN). To date, research on AN has been limited as compared to other $A$. annua extracts. One report demonstrates that it is composed of several phenolics and terpenes, resulting in antimicrobial activity [8]. As noted previously, A. annua extracts have shown promise 
in promoting healthy skin. The novel AN oil described here is purified by rectification to obtain high boiling point components $\left(>90^{\circ} \mathrm{C}\right)$. Thus, when compared to the more common and well-studied water extracts of $A$. апnиa, this AN oil extract is more lipophilic suggesting its bioactive molecules will penetrate the skin more effectively. Moreover, A. annua water extracts contain chlorophyll, making it difficult to remove its color which serves as an impediment for topical formulation development. Conversely, AN oil extract, given the elevated process temperatures, contains significantly less impurities, consistent batch to batch quality and its lightyellow color makes it more amenable to be used in many different formulation types.

Here, we demonstrate for the first time that this new AN oil extract has a broad range of anti-inflammatory properties, reducing pro-inflammatory cytokines such as IL-6, IL-8 and thymic stromal lymphopoietin (TSLP), a key marker for itch. Moreover, we show AN oil extract upregulates filaggrin, a key protein in epidermal differentiation and expand our understanding of its antimicrobial activity, as it inhibits the growth of several microbes including $C$. acnes and $S$. aureus which play a critical role in the pathogenesis of acne and eczema. We characterize the composition of AN oil extract, reporting its most abundant bioactive molecules are of camphor, camphene, p-cymene, eucolyptol and D-limonene. Lastly, clinical results in human subjects demonstrate AN oil formulated at $1 \%$ in a gel and applied topically is well tolerated and effective in reducing blemishes and the appearance of redness in acne prone skin.

\section{Materials and methods}

\section{Reagents}

All chemicals were obtained from Sigma-Aldrich Co. (St Louis, MO) and ThermoFisher (Waltham, MA). Artemisia naphta (AN) oil extract was provided by KPC Pharmaceuticals, Inc.

\section{Artemisia naphta oil extraction}

A. аnnua leaves were heated with petroleum ether and resulting AN oil was filtered. Leaching solution was heated and evaporated to remove the petroleum ether. AN oil was distilled at $100-110{ }^{\circ} \mathrm{C}$ and then drained to obtain crude AN oil. Crude product of AN oil was placed in a refining tank, heated to $110{ }^{\circ} \mathrm{C}$ for $30 \mathrm{~min}$ and then cooled down. Silica gel was added to the oil, stirred for 30 minutes and then filtered to decolorize the AN oil extract.

\section{GC-MS analysis}

One gram sample of AN oil extract was diluted with ethanol to $10 \%$ for GCMS analysis. Separation was performed on an Agilent HP-5MS column ( $\left.30 \mathrm{~m}^{*} 0.25 \mathrm{~mm} * 0.25 \mu \mathrm{M}\right)$ at a flow rate of $1 \mathrm{~mL} / \mathrm{min}$, split ratio (20:1), eluant gas ( $\mathrm{He}>99.9 \%$ ), with a temperature program of $60^{\circ}, 230^{\circ}, 240^{\circ}, 280{ }^{\circ} \mathrm{C}$.
Electron ionization (ionization energy $=70 \mathrm{eV}$ ) was utilized with a scan range of 29-550 amu, solvent delay $2 \mathrm{~min}$ and MS temperature of $230^{\circ} \mathrm{C}$ for source and $150{ }^{\circ} \mathrm{C}$ for quadrupole. The total ion flow diagram of the sample was obtained and identified the various components. The relative content of each component in the sample was determined by peak area normalization.

\section{Antimicrobial assays}

All antimicrobial testing was performed by third party organizations - Hozan Laboratories and Guangdong Biocell Biotechnology (Shanghai, China). In short, Cutibacterium acnes strain (CICC 10312) was cultured in Brain Heart Infusion Agar (BHIA) under anaerobic conditions. AN oil extract was added to BHIA with final concentrations ranging from $0.1-50 \mathrm{mg} /$ $\mathrm{mL}$. A fixed $1 \mu \mathrm{l}$ of bacterial suspension was spread on agar plates and incubated for $48-72$ hours at $37^{\circ} \mathrm{C}$. Malassezia furfur $\left(\mathrm{ATCC}^{\circledR} 44344^{\mathrm{TM}}\right.$ ) was cultured in media under anaerobic conditions. AN oil extract was added to media with final concentrations ranging from $0.5-200 \mathrm{mg} / \mathrm{mL}$. A fixed $1 \mu \mathrm{l}$ of bacterial suspension was spread on agar plates and incubated for $48-72$ hours at $37{ }^{\circ} \mathrm{C}$. After incubation, plates were placed on a black, non-reflective background to read results. Staphylococcus aureus $\left(\mathrm{ATCC}^{\circledR} 6538^{\mathrm{TM}}\right.$ ) and Candida albicans $\left(\right.$ ATCC $^{\circledR} 10231^{\mathrm{TM}}$ ) was cultured in Nutrient Broth under aerobic conditions. AN oil extract was added to media with final concentrations ranging from $2.5-400 \mathrm{mg} / \mathrm{mL}$. A fixed $100 \mu \mathrm{l}$ of bacterial suspension was spread on agar plates and incubated for 48 hours at $37{ }^{\circ} \mathrm{C}$. For all strains tested, bacterial suspension was prepared with a concentration of $1 \times 10^{8} \mathrm{CFU} / \mathrm{mL} \sim 2 \times 10^{8} \mathrm{CFU} / \mathrm{mL}$ as the initial inoculum. Minimum inhibitory concentration was defined as the lowest concentration which inhibited visible growth of bacteria.

\section{Cell culture}

All in vitro cell work was performed by Shanghai Archgene Biotechnology Co. (China) and Signum Biosciences (USA). In short, Raw 264.7 mouse macrophage were purchased from the Cell Bank, Chinese Academy of Sciences (Cat. tCM13). Raw 264.7 macrophages were maintained in DMEM containing $10 \% \mathrm{FBS}$ at $37{ }^{\circ} \mathrm{C}$ with $5 \% \mathrm{CO}_{2}$. When cells reached $90 \%$ confluence, cells were seeded in 96-well plate at a density of $8 \times 10^{5}$ per mL. After 8 hours, cells were treated by different concentrations of samples and incubated for 24 hours. The cells were randomly divided into different groups and cotreated with LPS $(1 \mu \mathrm{g} / \mathrm{mL})$ and test materials based on maximum non-toxic concentrations determined by CCK-8 kit (Sigma-Aldrich Co, St. Louis, MO). Dexamethasone $(20 \mu \mathrm{M}$ or $80 \mu \mathrm{M}$ ) was used as positive anti-inflammatory control. After 72 hours, cell supernatants were collected for IL-6 ELISA measurements (eBioscience, San Diego, CA). Normal human epidermal keratinocytes (NHEKs) from neonatal donors were purchased from ThermoFisher (Waltham, MA) and cultured in vitro in EpiLife ${ }^{\circledR}$ medium supplemented with 
HKGS at normal cell culture conditions $\left(37^{\circ} \mathrm{C}, 95 \% \mathrm{v} / \mathrm{v}\right.$ air, $5 \% \mathrm{v} / \mathrm{v} \mathrm{CO}_{2}$ ) and randomly separated into treatment groups based on maximum non-toxic concentration determined by MTS kit (Promega, Madison, WI). After 48 hours, test and control materials were pre-treated in cell culture media and incubated for 1 hour at standard culture conditions $\left(37^{\circ} \mathrm{C}\right.$ and $5 \% \mathrm{CO}_{2}$ ). Later, treatment media was replaced with live S. aureus (ATCC ${ }^{\circledR} 29213^{\mathrm{TM}} ; 1 \times 10^{7}$ CFU/mL) in fresh EpiLife ${ }^{\circledR}$ medium with test materials and incubated for 24 hours. After incubation, culture medium supernatants were harvested and used to measure IL-8 (BD Biosciences, San Jose, CA) and TSLP (R\&D Systems, Minneapolis, MN) levels by ELISA. NHEKs were treated with IL-4/IL-13 (30 ng/mL) with or without samples for 72 hours based on maximum non-toxic concentration and cell supernatants were collected to measure IL-8 by ELISA.

\section{Gene expression}

Primary human keratinocytes were co-treated with samples and IL-4 and IL-13 (30 ng/mL) for 72 hours. Supernatant was discarded and cells were washed and collected for total RNA extraction. RNA was reverse transcripted to cDNA and FLG gene expression was measured by qRT-PCR. The data was analyzed by $2^{-\Delta \Delta \mathrm{Ct}}$ approach.

\section{Clinical study}

A clinical trial in healthy volunteers was conducted at Shanghai Kunyao Biotechnology Co, Ltd (China). This was a single-blinded study, with 13 subjects, age range from 18 to 40 years, all with acne prone skin (inflammatory dermatitis, including papules and pustules). The study was conducted according to the principles of Good Clinical Practice and followed the ethical principles of the Declaration of Helsinki. The study protocol was approved by the ethical review board of Shanghai Chicmax Cosmetic Co (IRB). Subjects reported to the testing facility for baseline screening at which time Informed Consent was obtained. All subjects completed and signed documentation stating, "commitment to voluntarily participate in the human trial test of cosmetics and to participate in the human trial period. Subjects used 1\% AN Gel (ad libitum) for 2 weeks applying it to blemishes on the face and skin parameters were collected by non-invasive skin tester, including VISIA ${ }^{\circledR}$-CR (Canfield Scientific, Fairfield, NJ) facial imaging and consumer self-assessment questionnaire at baseline, day 3, day 7 and day 14. Clinical trials were performed in compliance with the Clinical Trial Scheme, ICH guidelines on Quality Management Practices of Clinical Trials and all applicable laws and regulations.

\section{Statistical analysis}

For gene and protein expression analysis, samples were assayed in triplicate. Statistical significance was determined by Student's $t$-test comparisons test using $p$ - values less than 0.05 as significant difference. For the Clinical study, the paired one-way ANOVA was used to assess within-subject improvements over baseline.

\section{Results}

Characterization of Artemisia naphtha (AN) oil extract chemical composition

The typical extraction process of artemisinin from A. annua powder results in non-volatile compounds (including artemisinin) and byproduct volatile components such as AN. AN is mainly composed of terpenoid and phenolic hydroxyl functional groups such as eucalyptol, terpinen-4-ol, $\alpha$-terpinyl propionate and $\beta$-pinene [8]. Using this AN byproduct as a starting point, distillation and rectification steps are then performed at $110{ }^{\circ} \mathrm{C}$, followed by decolorization yielding a novel, purified AN oil extract. Utilizing GC-MS analysis, the chemical composition of our novel AN oil extract was characterized. We identified eucalyptol as a major component present at similar levels in both AN (5.2\%) [8] and our AN oil extract (5.6\%, Table 1). Eucalytpol (also referred to as 1,8-cineole) has a mint-like scent and in addition to its antibacterial activity it is also a strong skin penetration enhancer [9]. The most abundant compound found in AN oil extract is camphor (23.7\%, Table 1$)$, a terpenoid which has been reported to provide several benefits to skin including wound healing properties and anti-wrinkle activity [10]. Camphene (aka as $\beta$-pinene) and p-cymene are two additional terpenes present at $16.2 \%$ and $13.9 \%$ respectively in AN oil extract. Both those compounds have been previously shown to possess a wide range of activities including antioxidant, anti-inflammatory, pain relief [11], anti-bacterial and antifungal properties [12]. Moreover, with their antimicrobial activity, camphene and p-cymene are found in many different commercial essential oils used to treat skin diseases including eczema and acne [13]. Several other compounds that promote skin health were also identified in our AN oil extract and are listed in table 1.

\section{AN oil extract possesses antibacterial activity}

It has been previously demonstrated that $A$. annua extracts [2] and artemisia naphtha (AN) [8] possess antimicrobial activity against several microbes including $C$. acnes and $S$. aureus which play key roles in the pathogenesis of acne and atopic dermatitis [14,15]. Moreover, A. annua extracts

\begin{tabular}{|c|c|}
\hline Table 1: Chemical composition of AN oil extract. & \% of AN oil \\
\hline Compound & 23.7 \\
\hline camphor & 16.2 \\
\hline camphene & 13.9 \\
\hline p-cymene & 5.6 \\
\hline eucalyptol & 3.9 \\
\hline D-limonene & 3.7 \\
\hline 3-Aminopyrazole & 2.5 \\
\hline Carene & 2.2 \\
\hline Terpinolene & 1.8 \\
\hline tricyclene & 1.0 \\
\hline Cyclohexanone & 25.5 \\
\hline Other & \\
\hline *The combination of all components $<1 \%$ each. & \\
\hline
\end{tabular}


have also been shown to have strong anti-fungal activity [16]. To investigate AN oil extract's potential antibacterial and anti-fungal properties, we tested for its minimal inhibitory concentration (MIC) versus the previously mentioned skin bacteria, $C$. acnes and $S$. aureus, as well as two fungi found on skin - Malassezia furfur and Candida albicans. AN oil extract demonstrated antibacterial activity against both $C$. acnes and $S$. aureus, with MIC values of $10 \mathrm{mg} / \mathrm{ml}$ and $5.6 \mathrm{mg} / \mathrm{ml}$ respectively (Table 2). Similarly, AN oil extract also exhibited anti-fungal properties inhibiting $M$. furfur ( $\mathrm{MIC}=5 \mathrm{mg} / \mathrm{ml}$ ) and $C$. albicans (MIC $=10 \mathrm{mg} / \mathrm{ml}$ ). 4-Terpineol, commonly found in tee tree oil and known as an antimicrobial compound, was utilized as a positive control and was effective (Table 2).

AN oil extract inhibits LPS and S. aureus induced IL-6, IL-8 and TSLP release

C. acnes induces an increase in IL-6, IL-8 and other proinflammatory cytokines in keratinocytes and macrophages through activation of Toll-like receptor 2 (TLR2) and Tolllike receptor 4 (TLR4) [17]. Since AN oil extract inhibits

\begin{tabular}{|c|c|c|}
\hline \multicolumn{1}{|c|}{ Table 2: Antimicrobial Activity of AN oil extract. } \\
\hline Organism & $\begin{array}{c}\text { AN oil extract MIC } \\
\mathbf{m g} / \mathbf{m l}(\%)\end{array}$ & $\begin{array}{c}\text { 4-Terpineol MIC } \\
\mathbf{m g} / \mathbf{m l}(\%)\end{array}$ \\
\hline C. acnes (CICC10312) & $10(1 \%)$ & $10(1 \%)$ \\
\hline S. aureus (ATCC 6538) & $5.6(0.56 \%)$ & $2.5(0.25 \%)$ \\
\hline M. furfur (ATCC44344) & $5(0.5 \%)$ & $0.5(0.05 \%)$ \\
\hline C. albicans (ATCC 1031) & $10(1 \%)$ & $2.5(0.25 \%)$ \\
\hline
\end{tabular}

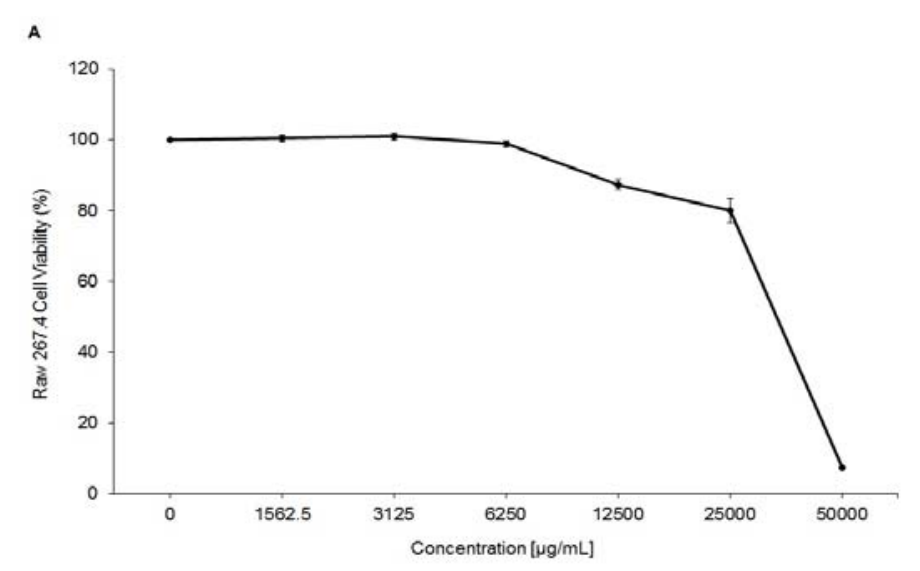

C. acnes growth, we want to begin to explore whether it could also inhibit TLR-induced inflammation. Lipopolysaccharide (LPS) triggers cytokine production through TLR4, which shares downstream signaling pathways with TLR2. Cell viability studies were performed with AN oil extract and Raw 264.7 macrophages to determine the highest nontoxic dose which was $25,000 \mu \mathrm{g} / \mathrm{mL}(2.5 \%)$ AN oil extract (Supplemental Data, Figure S1 a). Our results show that AN oil extract strongly reduces LPS-TLR4-induced IL-6 release from Raw 264.7 macrophages in a dose dependent manner (Figure 1). Dexamethasone, a corticosteroid and potent antiinflammatory, was used as a positive control and effectively reduced IL-6 production (Figure 1).

As is the case with $C$. acnes playing a key role in the presentation of acne on the skin, $S$. aureus plays a critical role in the pathogenesis of eczema, dry skin, sensitive skin, and itch [18,19]. Given AN oil extract demonstrated antibacterial activity against $S$. aureus, we investigated if it could also block $S$. aureus induced inflammation and itch signaling. Cell viability studies were performed with AN oil extract and normal human epidermal keratinocytes (NHEKs) to determine the highest non-toxic dose which was $100 \mu \mathrm{g} / \mathrm{mL}$ $(0.01 \%)$ AN oil extract (Supplemental Data, Figure S1b). Our results show that AN oil extract dose-dependently inhibits $S$. aureus induced IL-8 release from NHEKs with an $\mathrm{IC}_{50}=0.2$ $\mathrm{ng} / \mathrm{mL}(0.00000002 \%)$ (Figure 2a). Moreover, AN oil extract
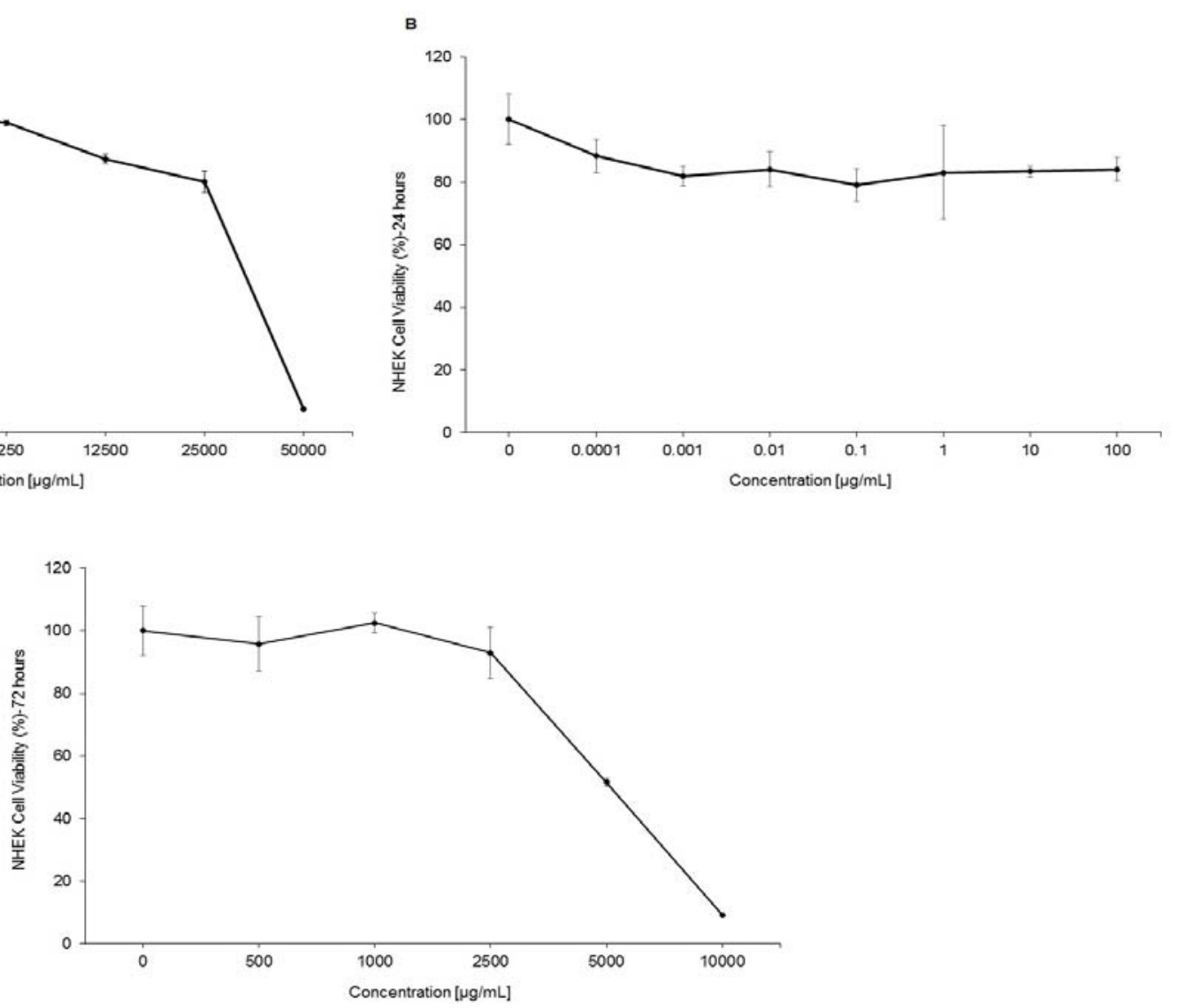
also reduces the production of thymic stromal lymphopoietin (TSLP) IC $_{50}=60 \mu \mathrm{g} / \mathrm{mL}(0.0006 \%$ ) (Figure $2 \mathrm{~b}$ ), a key protein that directly stimulates sensory neurons triggering pruritus [20].

\section{AN oil extract modulates IL-4/IL-13 induced keratinocyte differentiation and inflammation}

Th2 cytokines such as IL-4 and IL-13 play a central role in activating inflammatory pathways and impairing epidermal barrier function in eczema, sensitive skin and other skin allergic reactions [21]. Building upon the positive results of AN oil extract versus $S$. aureus induced inflammation and itch, we sought to study the effects of this extract against IL-4/IL13 induced inflammation and keratinocyte differentiation. Filaggrin (FLG) is a key protein in epidermal differentiation and contributes to the structural and functional integrity of the stratum corneum [22]. Our results show NHEKs downregulate FLG gene expression after IL-4/IL-13 treatment, suggesting Th2 activation negatively impacts skin barrier function. Cell viability studies were performed with AN oil extract and NHEKs to determine the highest non-toxic

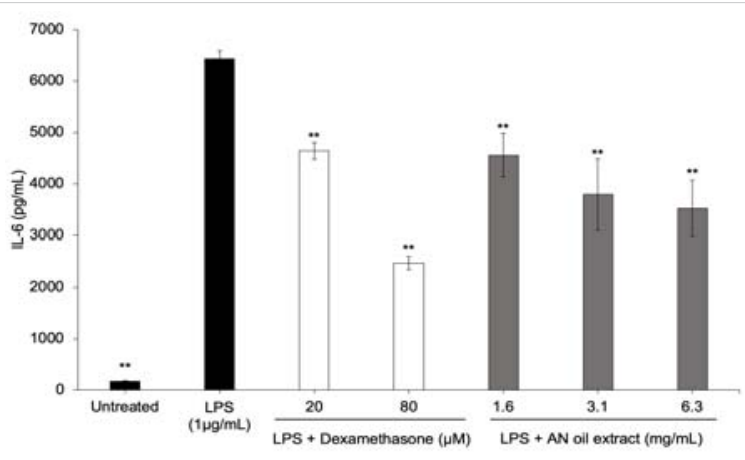

Figure 1: AN oil extract has anti-inflammatory activity inhibiting LPS-TLR4 proinflammatory IL-6 production. Raw 247.4 cells were co-incubated with LPS $(1 \mu \mathrm{g} / \mathrm{mL})$ and AN oil ( $0.16-0.63 \%)$ or dexamethasone $(20-80 \mu \mathrm{M})$ for 24 hours. Pro-inflammatory cytokine (IL-6) was measured from media supernatants by ELISA. The data represent the mean \pm stdev of cumulative data from three independent experiments. ${ }^{* *} p \leq 0.01$ indicates a statistically significant difference compared to LPS-only group. dose under these test conditions which was $2500 \mu \mathrm{g} / \mathrm{mL}$ (0.25\%) AN oil extract (Supplemental Data, Figure S1c). Interestingly, when $\mathrm{AN}$ oil extract was added together with IL-4/IL-13, FLG expression was significantly upregulated in a dose-dependent manner (Figure 3a). Thus, suggesting AN oil extract plays a protective role against Th2 activation and skin barrier function. As noted above, IL-4/IL-13 also activate inflammatory pathways, therefore we investigated whether AN oil extract could abrogate this effect. Results demonstrate that NHEKs treated with IL-4/IL-13 had significant induction of pro-inflammatory cytokine IL-8, and AN oil extract was able to significantly reduce IL-8 secretion in a dose dependent manner (Figure 3b).

\section{AN oil extract reduces blemishes and the appearance of redness in acne prone skin}

In view of AN oil extract's in vitro anti-bacterial activity vs C. acnes and its ability to reduce TLR4 induced inflammation, one of several TLRs important in promoting inflammatory acne, we sought to determine its tolerability and activity in human subjects. $1 \%$ AN oil extract was first tested clinically in a 72-hour human patch test (30 subjects) and was found to cause no skin sensitization or irritation and no erythema was observed (data not shown). Given this result, a 1\% AN oil extract in a topical gel ( $1 \% \mathrm{AN}$ Gel) was then tested in a single center (13 subjects) to evaluate its ability to remove blemishes in subjects with acne prone skin. No adverse effects were reported, indicating again that $1 \% \mathrm{AN}$ Gel was well tolerated. Visia-CR facial imaging showed reduction in the number and size of blemishes over 14 days, with some subjects showing improvement after only 3 days of application (Figure 4). Utilizing Canfield's RBX ${ }^{\circledR}$ technology to separate red and brown area maps to visualize inflammation, we observed a reduction in blemishes (Figure 4). Self-perception questionnaires completed by the subjects demonstrated that $85 \%$ of the users felt they noticed elimination of acne blemishes on their

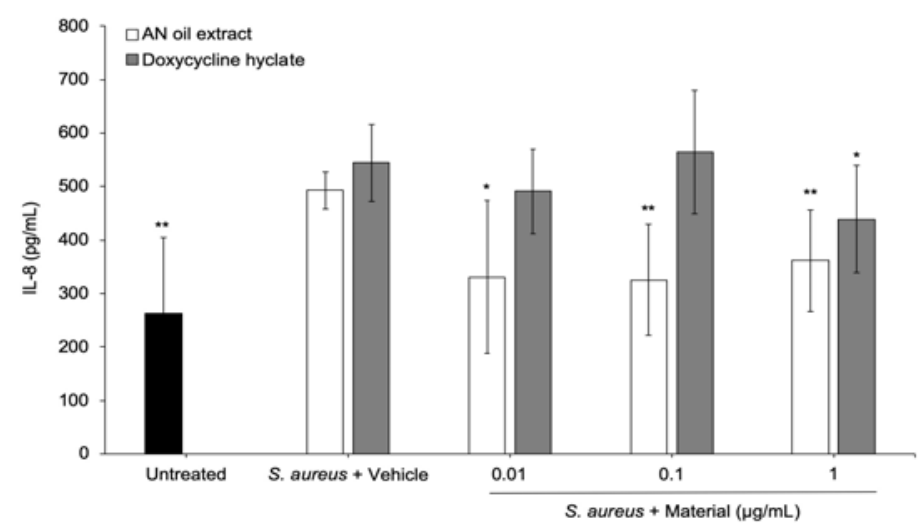

B

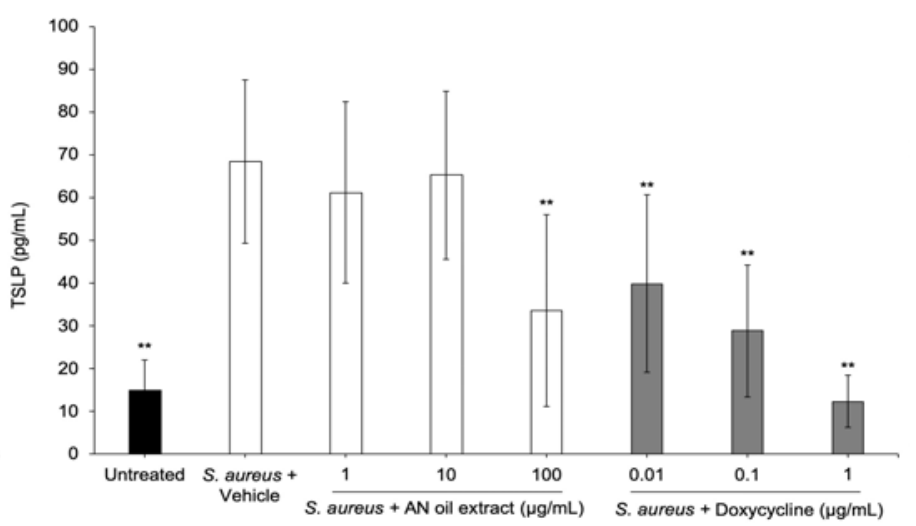

Figure 2: AN oil extract has anti-inflammatory activity blocking bacteria-induced pro-inflammatory cytokine release. NHEKs were incubated with S. aureus $\left(1 \times 10^{7} \mathrm{CFU} / \mathrm{mL}\right)$ and AN oil $(0.01-1 \mu \mathrm{g} / \mathrm{mL})$ or doxycycline hyclate $(0.01-1 \mu \mathrm{g} / \mathrm{mL})$ for 24 hours. (A) IL-8 or (B) TSLP levels were measured from media supernatants by ELISA. The data represent the mean \pm stdev of cumulative data from three independent experiments. ${ }^{*} p<0.05 ;{ }^{* *} p \leq 0.01$ indicates a statistically significant difference compared to $S$. aureus + vehicle group. 

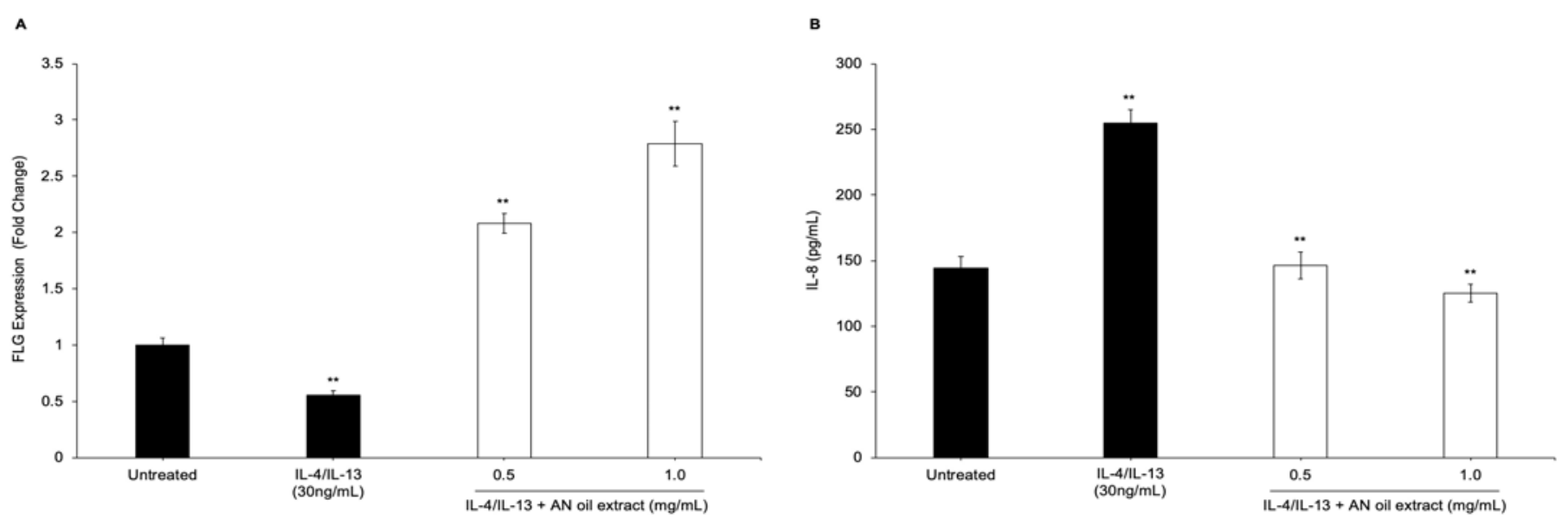

Figure 3: AN oil extract increases filaggrin gene expression and decreases Th2 cytokine-induced IL-8 cytokine production in a dose-dependent manner. NHEKs were treated with AN oil $(0.05-0.1 \%)$ for 72 hours. (A) Cells were harvested for gene expression analysis. The level of gene expression of filaggrin (FLG) was quantitated by qPCR normalized to YWHAZ gene. (B) Pro-inflammatory cytokine (IL-8) was measured from media supernatants by ELISA. The data represent the mean \pm stdev of cumulative data from three independent experiments. ${ }^{* *} p \leq 0.01$ indicates a statistically significant difference compared to untreated cells.
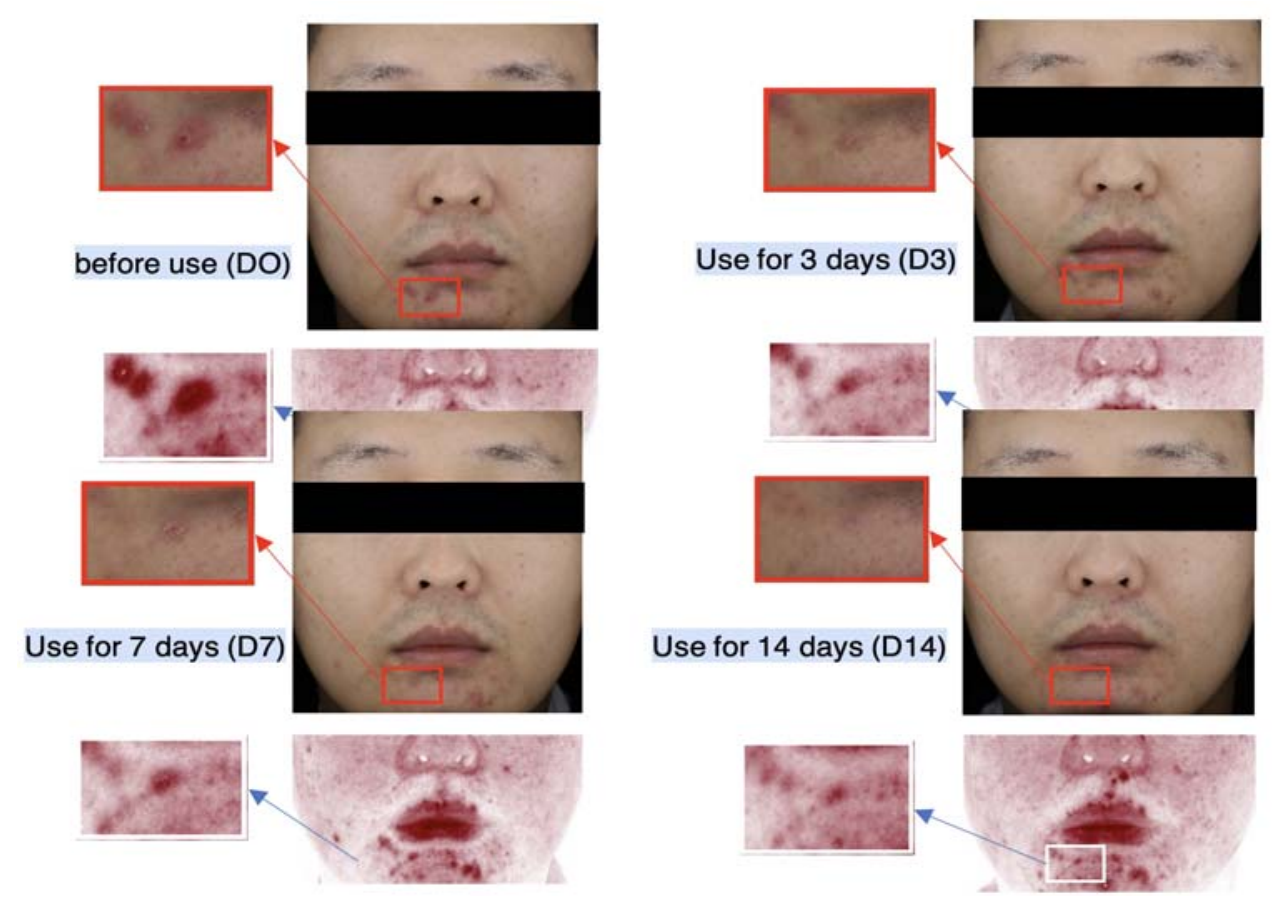

Figure 4: AN oil extract in a topical gel was tested to evaluate its ability to remove blemishes in subjects with acne prone skin. The subject applied $1 \%$ AN gel topically. Canfield's RBX ${ }^{\oplus}$ technology was utilized to separate red and brown area maps. Acne and erythema were observed to be reduced utilizing this imaging technology. Red zone area and Rate of change: D0(92.82 $\left.\mathrm{mm}^{2}\right)$, D3(26.59 mm², $\left.-71.36 \%\right), \mathrm{D} 7\left(22.50 \mathrm{~mm}^{2},-75.76 \%\right)$, D14(19.73 $\left.\mathrm{mm}^{2},-78.85 \%\right)$.

face after 7 days (Table 3). Moreover, 77\% of subjects using $1 \%$ AN Gel reported a reduction in the appearance of redness and swelling. Interestingly, 54\% - 62\% also reported relief in discomfort and itch (Table 3). Altogether, this proof-ofconcept clinical study demonstrates AN oil extract possesses promising skin protecting properties that should be studied further.

\section{Discussion}

We demonstrate in this study that AN oil, an extract of artemisia naphtha, which is normally disregarded when processing A. annua for artemisinin, provides several benefits to skin. Based on the data presented here, this can best be explained by the chemical composition of this novel AN oil extract. Via GC-MS analysis we identified AN oil extract contains several terpenes including its three most abundant compounds, camphor ( 24\%), Camphene (aka as $\beta$-pinene) $(\sim 16 \%)$ and p-cymene $(\sim 14 \%)$, all of which have been previously reported to possess skin protecting properties such as wound healing, anti-aging, anti-oxidant and anti-inflammatory properties $[10,11]$. The unique blend of skin actives identified in AN oil extract are potentially more 
effective when applied topically to skin given the presence of eucalyptol $(\sim 6 \%)$, which is a known penetration enhancer [9]. Eucalyptol may help drive AN oil extract's bioactive molecules into the skin and maximize AN oil extract's effectiveness. Altogether, producing this AN oil extract from material that was previously a byproduct provides a "win-win" scenario where we reduce the environmental footprint of artemisinin extraction from $A$. annua and develop an effective oil extract for skin.

Artemisinin has been studied extensively given its pharmaceutical applications, however it is currently banned in China for use as a cosmetic ingredient, as it is a pharmaceutical agent used to treat disease. However, the plant $A$. annua is a commonly used Traditional Chinese Medicine and approved for cosmetic use. To this end, the use of $A$. annua extracts have recently started to emerge in cosmetic products. In a study published this past year, $\mathrm{Yu}$, et al. demonstrate that $A$. annua extract in a human clinical trial increases hydration, and reduces both transepidermal water loss (TEWL) and inflammation, signaling effectiveness for sensitive skin [7]. Building on these encouraging results and the goal to make use of a rarely used byproduct to utilize the many bioactive molecules present in $A$. annua, we sought to develop the first artemisinin-free $A$. annua extract for cosmetic use. From a manufacturing and formulation perspective, current $A$. annua water extracts present a couple challenges that are difficult to overcome. First, they contain chlorophyll, making its color difficult to remove and limiting its ability to be used in a wide range of formulation types. Conversely, AN oil extract's light-yellow color is much more amenable to a variety of formulation types including white creams and face masks. Moreover, the process of making AN oil yields an extract with higher purity and less batch-to-batch variability than $A$. annua water extracts. Here we demonstrate for the first time, the use of a novel artemisia naphta derived oil extract when applied topically is effective in reducing blemishes and redness in acne prone skin (Figure 4, Table 3). This proof-of-concept clinical study demonstrates that AN oil extract formulated at $1 \%$ in a gel is not only well-tolerated, but effective. Moreover, after 7 days, $62 \%$ of subjects reported a reduction in pain and itch, suggesting this effect could be due in part to inhibition of TSLP, as shown in Figure 2b. AN oil extract's encouraging in vitro profile demonstrating antimicrobial properties (Table 2) paired with anti-inflammatory activity against TLR4, S. aureus and Th2 response, suggests AN oil extract may have several applications in skin care including ameliorating acne and

\begin{tabular}{|c|c|c|c|}
\hline Table 3: Clinical Self-Perception Questionnaire Results. & & \\
\hline Questions & Day 3 & Day 7 & Day 14 \\
\hline Quickly reduces redness and swelling & $62 \%$ & $69 \%$ & $77 \%$ \\
\hline Eliminates initial blemishes & $54 \%$ & $85 \%$ & $62 \%$ \\
\hline Reduces number of blemishes & ND & $46 \%$ & $62 \%$ \\
\hline Can relieve discomfort and itch in area of blemishes & $54 \%$ & $62 \%$ & $54 \%$ \\
\hline Effectively prevents the recurrence of blemishes & ND & $46 \%$ & $46 \%$ \\
\hline
\end{tabular}

sensitive skin. This was also observed in the proof-of-concept clinical study where utilizing $\mathrm{RBX}^{\circledR}$ imaging to visualize inflammation, we see a reduction of red area map images, suggesting AN oil extract's anti-inflammatory properties are effective when applied topically. AN oil extract's potential skin protecting properties are further buttressed by the in vitro data presented here showing it may also be able to promote healthy skin barrier function by upregulating filaggrin (Figure 3a). Our next goals are to investigate further the role AN oil extract plays in modulating TLR signaling, how it compares in activity to artemisinin and to run vehiclecontrolled clinicals to study its potential benefits in subjects with sensitive and acne prone skin.

\section{Conclusion}

We demonstrate here the utilization of an $A$. annua extraction byproduct as a source, and subsequent discovery of a novel AN oil extract that is enriched for numerous terpenes that provide several benefits to the skin. AN oil extract possesses antimicrobial activity against several microorganisms including $C$. acnes and $S$. aureus, which are both implicated in acne and eczema. Moreover, AN oil extract is shown to block TLR4, S. aureus, and IL-4/IL-13 induced inflammation and upregulate filaggrin, all which have been previously shown to be critical in acne and eczema pathogenesis. Lastly, in a human clinical study, topical application of a $1 \% \mathrm{AN} \mathrm{Gel,} \mathrm{is} \mathrm{reported} \mathrm{to} \mathrm{reduce} \mathrm{blemishes}$ and redness in subjects. Altogether the in vitro and clinical data presented here suggest AN oil extract will be effective for those with sensitive and/or acne prone skin.

\section{References}

1. Mueller MS, Karhagomba IB, Hirt HM, Wemakor E. The potential of Artemisia annua L. as a locally produced remedy for malaria in the tropics: agricultural, chemical and clinical aspects. J Ethnopharmacol. 2000; 73: 487-493.

PubMed: https://pubmed.ncbi.nlm.nih.gov/11091003/

2. Septembre-Malaterre A, Rakoto ML, Marodon C, Bedoui Y, Jessica Nakab et al. Artemisia annua, a Traditional Plant Brought to Light. Int J Mol Sci. 2020; 21: 4986.

PubMed: https://pubmed.ncbi.nlm.nih.gov/32679734/

3. Li T, Chen H, Wei N, Mei X, Zhang S, et al. Anti-inflammatory and immunomodulatory mechanisms of artemisinin on contact hypersensitivity. Int Immunopharmacol. 2012; 12: 144-150. PubMed: https://pubmed.ncbi.nlm.nih.gov/22122827/

4. Xue X, Dong Z, Deng Y, Yin S, Wang P, et al. [Dihydroartemisinin alleviates atopic dermatitis in mice by inhibiting mast cell infiltration]. Nan Fang Yi Ke Da Xue Xue Bao. 2020; 40: 1480-1487. PubMed: https://pubmed.ncbi.nlm.nih.gov/33118501/

5. Li YJ, Guo Y, Yang Q Weng XG, Yang L, et al. Flavonoids casticin and chrysosplenol D from Artemisia annua L. inhibit inflammation in vitro and in vivo. Toxicol Appl Pharmacol. 2015; 286: 151-158. PubMed: https://pubmed.ncbi.nlm.nih.gov/25891417/

6. Ciftci ON, Cahyadi J, Guigard SE, Saldaña MDA. Optimization of artemisinin extraction from Artemisia annua L. with supercritical carbon dioxide + ethanol using response surface methodology. Electrophoresis. 2018.

PubMed: https://pubmed.ncbi.nlm.nih.gov/29756212/ 
7. Yu J, Wang G, Jiang N. Study on the repairing effect of cosmetics containing artemisia annua on sensitive skin. J Cosmetics Dermatol Sci Appli. 2020; 10: 8-19.

8. Wu C, Yan Y, Wang Y, Sun P, Qi R. Antibacterial epoxy composites with addition of natural Artemisisa annua waste. e-Polymers. 2020; 20: 262-271.

9. Saify ZS, Ahsan O, Dayo A. Cineole as skin penetration enhancer. Pak J Pharm Sci. 2000; 13: 29-32.

PubMed: https://pubmed.ncbi.nlm.nih.gov/16414836/

10. Tran TA, Ho MT, Song YW, Cho M, Cho SK. Camphor Induces Proliferative and Anti-senescence Activities in Human Primary Dermal Fibroblasts and Inhibits UV-Induced Wrinkle Formation in Mouse Skin. Phytother Res. 2015; 29: 1917-1925.

PubMed: https://pubmed.ncbi.nlm.nih.gov/26458283/

11. Quintans-Junior L, Moreira JCF, Pasquali MAB, Rabie SMS, Pires AS, et al. Antinociceptive Activity and Redox Profile of the Monoterpenes $(+)$-Camphene, p-Cymene, and Geranyl Acetate in Experimental Models. ISRN Toxicol. 2013; 2013: 459530.

PubMed: https://pubmed.ncbi.nlm.nih.gov/23724298/

12. Marchese A, Arciola CR, Barbieri R, Silva AS, Nabavi SF, et al. Update on Monoterpenes as Antimicrobial Agents: A Particular Focus on p-Cymene. Materials (Basel). 2017; 10: 947.

PubMed: https://pubmed.ncbi.nlm.nih.gov/28809799/

13. Luna A, Tarifa MF, Fernandez ME, Caliva JE, Pellegrini S, et al. Thymol, alpha tocopherol, and ascorbyl palmitate supplementation as growth enhancers for broiler chickens. Poult Sci. 2019; 98: 1012-1016. PubMed: https://pubmed.ncbi.nlm.nih.gov/30165460/

14. Scholz CFP, Kilian M. The natural history of cutaneous propionibacteria, and reclassification of selected species within the genus Propionibacterium to the proposed novel genera Acidipropionibacterium gen. nov, Cutibacterium gen. nov. and Pseudopropionibacterium gen. nov. Int J Syst Evol Microbiol. 2016; 66: 4422-4432.

PubMed: https://pubmed.ncbi.nlm.nih.gov/27488827/
15. Hauser C, Wuethrich B, Matter L, Wilhelm JA, Schopfer K. The immune response to $S$. aureus in atopic dermatitis. Acta Derm Venereol Suppl (Stockh). 1985; 114: 101-104.

PubMed: https://pubmed.ncbi.nlm.nih.gov/3859157/

16. Liu CH, Zou WX, Lu H, Tan RX. Antifungal activity of Artemisia annua endophyte cultures against phytopathogenic fungi. J Biotechnol. 2001; 88: 277-282.

PubMed: https://pubmed.ncbi.nlm.nih.gov/11434973/

17. Jugeau S, Tenaud I, Knol AC, Jarrousse V, Quereux G, et al. Induction of toll-like receptors by Propionibacterium acnes. $\mathrm{Br} J$ Dermatol. 2005; 153: 1105-1113.

PubMed: https://pubmed.ncbi.nlm.nih.gov/16307644/

18. Gong JQ, Lin L, Lin T, Hao F, Zeng FQ, et al. Skin colonization by Staphylococcus aureus in patients with eczema and atopic dermatitis and relevant combined topical therapy: a double-blind multicentre randomized controlled trial. $\mathrm{Br} \mathrm{J}$ Dermatol. 2006; 155: 680-687. PubMed: https://pubmed.ncbi.nlm.nih.gov/16965415/

19. Blicharz L, Usarek P, Młynarczyk G, Skowroński K, Rudnicka L, et al Is Itch Intensity in Atopic Dermatitis Associated with Skin Colonization by Staphylococcus aureus? Indian J Dermatol. 2020; 65: 17-21. PubMed: https://pubmed.ncbi.nlm.nih.gov/32029934/

20. Turner MJ, Zhou B. A new itch to scratch for TSLP. Trends Immunol. 2014; 35: 49-50.

PubMed: https://pubmed.ncbi.nlm.nih.gov/24412411/

21. Leung DY, Boguniewicz M, Howell MD, Nomura I, Hamid QA. New insights into atopic dermatitis. J Clin Invest, 2004. 113: 651-657. PubMed: https://pubmed.ncbi.nlm.nih.gov/14991059/

22. Levin J, Friedlander SF, Del Rosso JQ. Atopic dermatitis and the stratum corneum: part 1: the role of filaggrin in the stratum corneum barrier and atopic skin. J Clin Aesthet Dermatol. 2013; 6: 16-22. PubMed: https://pubmed.ncbi.nlm.nih.gov/24155988/ 